\title{
Short-Duration Hypothermia Induction in Rats using Models for Studies examining Clinical Relevance and Mechanisms
}

\author{
Daniel Omileke ${ }^{1}$, Steven BothwelI ${ }^{1}$, Daniel J. Beard ${ }^{1}$, Nikolce Mackovski ${ }^{1}$, Sara Azarpeykan ${ }^{1}$, Kirsten Coupland ${ }^{1}$, Adjanie \\ Patabendige $^{1}$, Neil Spratt $^{1,2}$ \\ ${ }^{1}$ School of Biomedical Sciences and Pharmacy, University of Newcastle ${ }^{2}$ Department of Neurology, John Hunter Hospital, Hunter New England Local \\ Health District
}

\section{Corresponding Author}

Neil Spratt

neil.spratt@newcastle.edu.au

\section{Citation}

Omileke, D., Bothwell, S., Beard, D.J., Mackovski, N., Azarpeykan, S., Coupland, K., Patabendige, A., Spratt, N. Short-Duration Hypothermia Induction in Rats using Models for Studies examining Clinical Relevance and Mechanisms. J. Vis. Exp. (169), e62325, doi:10.3791/62325 (2021).

\section{Date Published}

March 3, 2021

DOI

$10.3791 / 62325$

URL

jove.com/video/62325

\section{Abstract}

Therapeutic hypothermia $(\mathrm{TH})$ is a powerful neuroprotective strategy that has provided robust evidence for neuroprotection in pre-clinical studies of neurological disorders. Despite strong pre-clinical evidence, TH has not shown efficacy in clinical trials of most neurological disorders. The only successful trials employing therapeutic hypothermia were related to cardiac arrest in adults and hypoxic ischemic injury in neonates. Further investigations into the parameters of its use, and study design comparisons between pre-clinical and clinical studies, are warranted. This article demonstrates two methods of short-duration hypothermia induction. The first method allows for rapid hypothermia induction in rats using ethanol spray and fans. This method works by cooling the skin, which has been less commonly used in clinical trials and may have different physiological effects. Cooling is much more rapid with this technique than is achievable in human patients due to differences in surface area to volume ratio. Along with this, a second method is also presented, which allows for a clinically achievable cooling rate for short-duration hypothermia. This method is easy to implement, reproducible and does not require active skin cooling.

\section{Introduction}

$\mathrm{TH}$ is the practice of cooling body or brain temperature in order to preserve the viability and function of the organ/ system ${ }^{1,2}$. The role of hypothermia in neuroprotection has been investigated and has shown benefits in a range of preclinical models of neurological diseases such as stroke ${ }^{3}$, subarachnoid hemorrhage ${ }^{4}$, and traumatic brain injury ${ }^{5}$. In terms of clinical applications, TH has shown efficacy in patients post-cardiac arrest and in neonatal hypoxic-ischemic injury ${ }^{6}$.

$\mathrm{TH}$ induction is achieved using either surface or endovascular cooling methods. The majority of pre-clinical hypothermia studies perform surface cooling by applying water or ethanol 
to the animal's fur, or by using a cooling blanket to achieve target temperature ${ }^{1}$. In humans, systemic surface cooling is achieved by using ice packs and cooling blankets ${ }^{7,8}$. More rapid cooling has been shown in patients using endovascular methods, which couple an induction infusion of cold saline through an intravenous or intra-arterial catheter, with the placement of an endovascular cooling device within the inferior vena cava ${ }^{9,10}$. For example, a moderate target temperature of $33{ }^{\circ} \mathrm{C}$ can be reached in $1.5 \mathrm{~h}$ with endovascular cooling compared to 3-4 $\mathrm{h}$ with surface cooling in patients ${ }^{11}$. The endovascular approach has also become more popular in recent years because it has been reported to reduce some of the side effects seen in systemic surface cooling, such as shivering ${ }^{12,13}$. The European multicenter, randomized phase III clinical trial of hypothermia for ischemic stroke (EUROHYP-1) used mostly surface cooling ${ }^{14}$. Results recently published from this trial showed that shivering was a major complication and might have limited the ability to achieve the target temperature ${ }^{10}$. The shivering response is known to be primarily driven by skin temperature. Some efforts have been made to develop a rodent endovascular cooling method ${ }^{15}$, but the highly invasive nature of the technique compared to that used in humans, risks confounding any results obtained from that model.

Temperature is the key modulator of biological processes in the body and is tightly regulated by homeostasis. Therefore, any manipulation of the body temperature can have associated risks. Cooling duration is a factor that may have limited the success of hypothermia clinical trials. These trials use a long-duration cooling method, with many maintaining hypothermia from 24-72 $\mathrm{h}^{11}$. This extended duration poses a risk for infection during the cooling protocol. Pneumonia is the most common complication from hypothermia, affecting between $40-50 \%$ of patients who undergo the procedure ${ }^{13}$. This is in contrast to what is normally seen in animal studies of hypothermia where a short-duration paradigm is used (1-6 $h)^{3}$. The success of these pre-clinical animal studies will likely result in the adaptation of short-duration hypothermia for the use in clinical trials. As a result, it is necessary to have an animal model of short-duration hypothermia that resembles the cooling rates of future clinical trials. Further details pertaining to other temperature parameters and the validity of short-duration hypothermia have been discussed in several review articles ${ }^{1,16,17,18}$.

Demonstrated here is a gradual model of cooling that is more clinically achievable than current experimental hypothermia models. This novel method has a much slower rate of cooling and therefore, the time to target temperature is closer to the range of those seen in clinical trials of hypothermia ${ }^{11}$. It also avoids direct surface cooling, which has specific physiological effects, and may, therefore, be more comparable to endovascular cooling, which has been the most commonly used cooling method in clinical trials ${ }^{9,12}$. This model allows animals to be cooled gradually over $2 \mathrm{~h}$ followed by a short period of maintenance at target temperature. Additionally, the rapid cooling short-duration hypothermia method ${ }^{19}$ is also demonstrated. The fastcooling method allows target temperature to be achieved rapidly after hypothermia onset. While this approach is not as clinically relevant as the gradual cooling method, it is useful for studies that aim to explore the mechanisms of hypothermia neuroprotection to potentially mimic its powerful neuroprotective effects pharmacologically. This method also has potential applications outside of neuroscience and could be adapted to any number of pre-clinical studies. Another advantage of both methods compared to other approaches is that they are inexpensive and do not require 
specialist equipment. Finally, this protocol also demonstrates implantation of temperature dataloggers, since post-operative warming and monitoring thereof are important to prevent inadvertent post-operative hypothermia, with its potential to confound study results 20 .

\section{Protocol}

All experimental procedures were in accordance with the Australian Code of Practice for the Care and Use of Animals for Scientific Purposes and were approved by the Animal Care and Ethics Committee of the University of Newcastle (A-2013-343 and A-2020-003). In addition to the hypothermia induction methods described below, the following protocols are routinely done in conjunction with hypothermia: femoral line cannulation to monitor blood pressure and heart rate ${ }^{21}$, and experimental stroke ${ }^{22}$.

\section{Datalogger implantation}

NOTE: The datalogger device used in this protocol was not capable of providing real time read-out of body temperature. Read out is possible once the datalogger has been removed from the animal and connected back to the computer. As a result, the rectal temperature probe is used to provide real time information during the cooling and rewarming process. Additionally, the rectal probe is also vital to this method because the surgical heat mat on which the animal is placed during the procedure is regulated by the rectal probe system. The datalogger also serves a valuable purpose of providing temperature data in freely moving, awake rats and is important for ensuring that normal body temperature is maintained after rewarming. Therefore, both temperaturemonitoring devices are important for this protocol.
1. Anesthetize 10-12-week-old male outbred Wistar rat with isoflurane ( $5 \%$ for induction and $2-2.5 \%$ for maintenance) in a $50 \% \mathrm{~N}_{2}$ and $50 \% \mathrm{O}_{2}$ mixture.

2. Following induction, place the rat in the prone position on a surgical heat mat.

3. Position the rat so that the nose is sitting in the nose cone. Secure the nose with surgical tape to ensure no gases are escaping.

4. Shave the fur from the lower right abdomen and inject the site subcutaneously with a local anesthetic, Bupivacaine $0.2 \mathrm{~mL}, 0.05 \%$.

5. Apply antiseptic solution to the freshly shaved region.

6. Using sterilized surgical tools, make a $2 \mathrm{~cm}$ longitudinal incision along the right abdominal region, proximal to the right thigh. Make the incision deep enough to expose the space at the ventral thigh crease.

7. Use hemostats and forceps to create a 'pocket' under the skin that is large enough to hold the device.

8. Insert the temperature monitoring datalogger device into the pocket and close the muscle and skin using 5-0 silk sutures. The subcutaneous method described here is preferred to the intra-peritoneal method as it is less invasive and allows for better recovery after the procedure.

9. Ensure datalogger and rectal probe are cross calibrated for temperature monitoring (see Discussion).

10. Ensure the datalogger is not resting against the animal's heat mat after insertion, as this will influence temperature readings. 

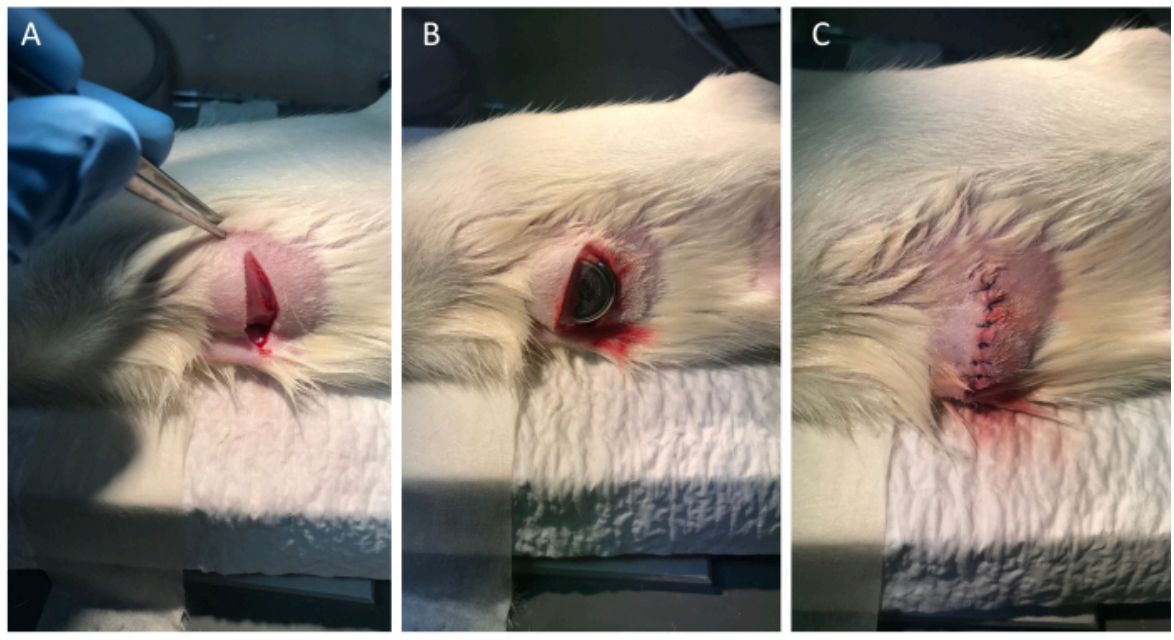

Figure 1: Implantation of datalogger device. (A) Panels left to right show an incision of approximately $2 \mathrm{~cm}$ being made on the right side of the lower abdomen of the rat. (B) Temperature monitoring datalogger was inserted subcutaneously into the pocket incision. (C) The incision was closed with nylon sutures. Please click here to view a larger version of this figure.

\section{Induction of active (fast) hypothermia for mechanistic studies}

1. Set up for hypothermia (see Figure 2). Place two retort stands with clamps at either side of the rat's body.

2. Attach a $60 \mathrm{~mm} 12 \mathrm{v} / 130 \mathrm{~mA}$ fan to each retort stand ensuring that the fans are aimed towards the lower back of the rat. The distance between the clamp and the rat is approximately $20 \mathrm{~cm}$. The fan used must have a speed of $4,000 \mathrm{rpm}$.

3. Have an animal heat lamp ready either at the side or on a third retort stand.

4. Commence hypothermia by adjusting the animal heat mat to the desired target temperature. In this example, $32.5{ }^{\circ} \mathrm{C}$ is the target temperature (3.75 on the temperature control unit).
5. Turn both fans on and apply three to four sprays of $70 \%$ ethanol (standard plastic spray bottle) on the lower back of the rat. Ruffle animal fur when spraying for faster cooling induction.

NOTE: Ethanol is used as a preferred solution over water because it has a faster rate of evaporation and therefore results in more rapid hypothermia induction.

6. Take care not to oversaturate the fur, since this can contribute to overshooting the target temperature.

NOTE: The fans will accelerate ethanol evaporation and the cooling process.

7. Allow short intervals between ethanol applications while keeping a close eye on the rectal temperature of the rat.

8. Cease any further application of ethanol once rectal temperature reaches within $1^{\circ} \mathrm{C}$ of target temperature.

9. Turn off both fans once the temperature has reached within $0.5^{\circ} \mathrm{C}$ of target temperature $\left(33^{\circ} \mathrm{C}\right.$ in this case $)$. 
NOTE: Turning off fans before the target temperature is reached helps prevent the rat from overcooling beyond required temperature.

10. Allow the temperature to drop to $32.5^{\circ} \mathrm{C}$.

1. If overcooling does occur, use animal heat lamp to mildly warm the animal back up to target. The assistance of one fan may be used to prevent a rewarming overshoot.

11. Once target temperature is reached and has stabilized, continue to monitor temperature. Temperature will usually remain very stable for the remainder of the hypothermia period without the need for spraying, use of fans or use of heat lamp.

12. To rewarm the animal at the end of hypothermia, adjust the heat mat temperature back to $37^{\circ} \mathrm{C}$ (6 on the temperature control unit used in this example) and allow the animal to thermoregulate over a 30 min period.

NOTE: Temperature settings on temperature control units may vary and it may, therefore, be necessary to determine settings for target hypothermia and normothermia on individual devices.

\section{Induction of clinically achievable gradual onset hypothermia without active skin cooling}

1. Achieve hypothermia by reducing the temperature of the core temperature regulated homeothermic heat mat in small increments to the required target temperature. In the illustrated example (Figure 3B), a $1{ }^{\circ} \mathrm{C}$ increment every 30 min was used.

2. Cool the animal to target temperature over the desired period ( $2 \mathrm{~h}$ in the example described). Once cooled, maintain at target for the desired interval. Usually, no further intervention is required if they are maintained on the homeothermic heat mat set for the desired target temperature.

3. No external cooling is necessary with this protocol as anesthesia prevents the normal regulation of core body temperature.

NOTE: Isoflurane requirements decline with hypothermia. In most animals, a starting isoflurane concentration of $2 \%$ can be reduced in $0.1 \%$ increments every $20-30 \mathrm{~min}$ to $1.5 \%$ to maintain stable respiratory rate (>50 breaths $/ \mathrm{min}$ ), heart rate and blood pressure, and preserve suppression of reflex responses.

4. To rewarm the animal after hypothermia, adjust the heat mat to allow the animal to rewarm over the desired interval. In the example, rewarming with a single adjustment to $37^{\circ} \mathrm{C}$ ( 6 on the $\mathrm{FHC}$ temperature control unit used in the example) was achieved over a $30 \mathrm{~min}$ period.

1. For longer-term studies that require animal recovery, keep animals in a cage that is placed half over a heat mat to allow the animal to thermoregulate and to avoid inadvertent post-operative hypothermia.

\section{Representative Results}

Figure $\mathbf{3 A}$ is a representation of how a Wistar rat responds to hypothermia using the rapid cooling approach. Hypothermia induction is achieved by the use of fans and $70 \%$ ethanol spray. Hypothermia to a target of $32.5{ }^{\circ} \mathrm{C}$ is achieved in 15 min. Care must be taken to ensure a delicate interplay between the use of the fans/ heat lamp and ethanol spray to maintain target temperature. As can be seen from Figure 3A, a slight temperature overshoot is observed, which may occur if cooling is not ceased from about $0.5^{\circ} \mathrm{C}$ above target 
temperature. Target is maintained and stabilized at the $30 \mathrm{~min}$ mark and rewarming is initiated at $1.5 \mathrm{~h}$.

Figure 3B shows the gradual protocol in which target temperature to $33{ }^{\circ} \mathrm{C}$ is reached at $2 \mathrm{~h}$ and maintained for $30 \mathrm{~min}$ before rewarming at $2.5 \mathrm{~h}$. Here, the temperature is adjusted in increments which prolongs the duration necessary to reach target temperature. Vertical dotted lines in both graphs represent the duration of cooling.
Figure 3A and Figure 3B are obtained from the datalogger device. At the start of the experiment, the datalogger is programmed to initiate recording prior to implantation. At the end of the experiment, the datalogger is removed from the animal and connected to the provided temperature reader via USB port. The software (e.g., eTemperature) reads and generates the data, which can then be exported to a spreadsheet software.
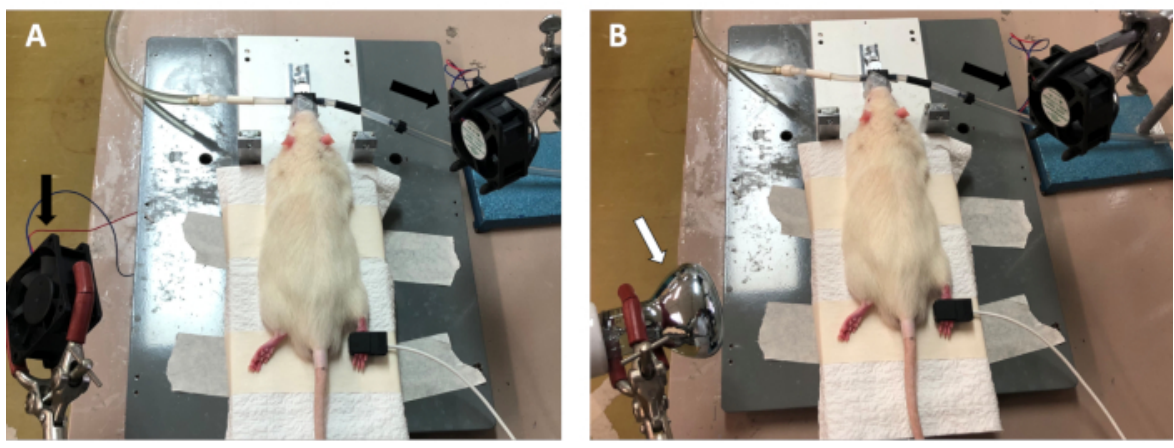

Figure 2: Set up of rapid cooling protocol. (A) Two fans (black arrow) were situated over the lower back region of the rat. At hypothermia initiation, both fans were turned on and ethanol spray was applied to the lower back. The combination of ethanol and the fan facilitates and accelerates hypothermia to rapidly achieve target temperature. (B) A heat lamp (white arrow) was used to prevent hypothermia overshoot. Once target temperature was reached, the heat lamp was used to prevent the rat core temperature from dropping any lower. Once target had stabilized, the heat lamp and/or remaining fan was turned off. Please click here to view a larger version of this figure. 
A

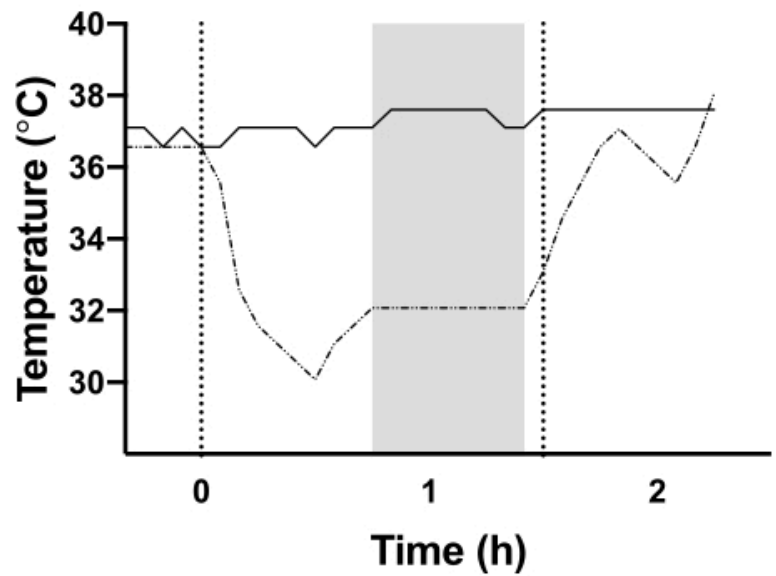

$32.5^{\circ} \mathrm{C}$

- Normothermia

\section{B}

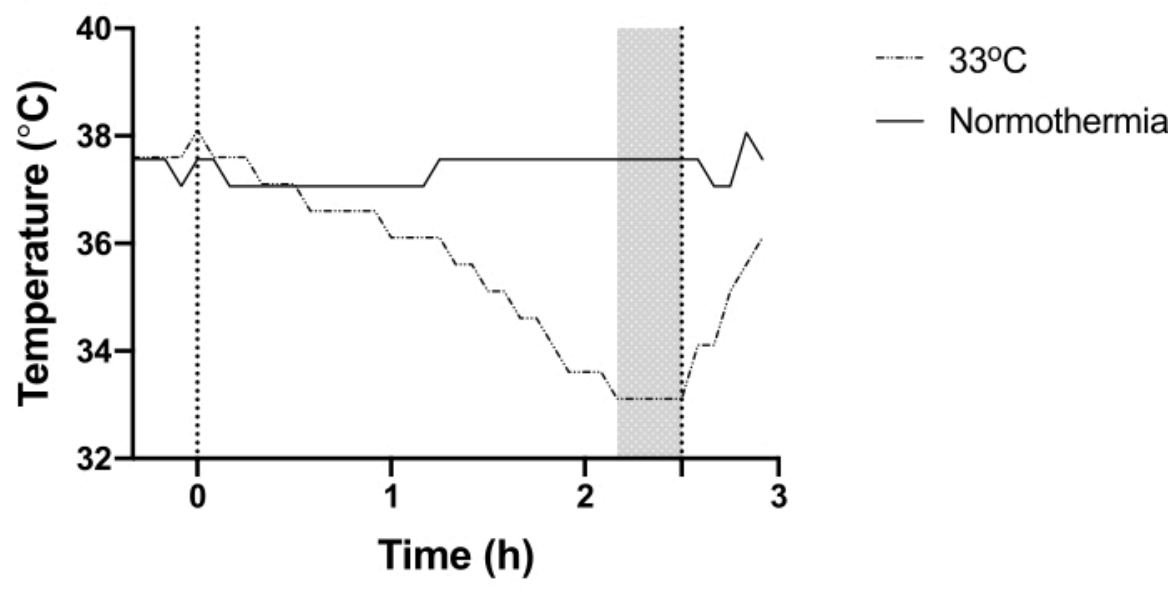

Figure 3: Hypothermia induction using active (A) and gradual (B) methods. (A) Target temperature was reached in 15 min using the active cooling process and was maintained for $60 \mathrm{~min}$ in the above example before the animal was rewarmed.

(B) Target temperature was reached in $2 \mathrm{~h}$ using the gradual cooling method and was maintained for 30 min before the animal was rewarmed. Shaded regions in both graphs represent time points in which target temperature was maintained. Dotted perpendicular lines in both graphs refer to the overall cooling duration. Please click here to view a larger version of this figure. 


\section{Discussion}

The procedures described here are easily implemented, noninvasive, and provide reliable and reproducible decreases in core body temperature to a desired target temperature.

There are several critical steps in the rapid cooling method which include the following. Do not oversaturate ethanol spray - care must be taken not to soak the animal in ethanol, as this will interfere with results. Monitor the animal during hypothermia induction- care must be taken to closely monitor animal responses to rapid hypothermia induction. A close watch of rectal temperature is important to ensure that temperature does not go below the desired target- if this happens, turn off the fans and allow the heat lamp to gently rewarm the animal back up to the required target.

In both methods, physiological monitoring is important to ensure appropriate adjustment of anesthetic dose. For prolonged cooling, inadequate anesthetic dose may prolong the duration of cooling. In this case, isoflurane concentration can be increased until an adequate cooling rate is achieved. Another critical step is the cross-calibration of temperature devices. When using a temperature probe regulated heat mat and a datalogger in the same experiment, it is best practice to cross-calibrate the datalogger with the rectal probe, in vivo, since there may be minor variations in the recorded temperature of the two devices.

These methods are suitable for studies that wish to explore the use of hypothermia as a potential treatment for neurological disorders. The specific aim of the study should dictate which method is used. Both methods can be classed as systemic surface cooling, however the second method does not require any active cooling. The gradual cooling model described above has important potential applications for the use of hypothermia in ischemic stroke treatment. Long- duration hypothermia and their resulting complications pose a challenge to elderly stroke patients. Moreover, the shivering response makes it difficult to achieve target temperature in some patients ${ }^{10}$. While anti-shivering medication can help to reduce the shivering response, short-duration gradual cooling could more effectively ameliorate the issue. Having a shorter cooling period is also likely to reduce the incidence of pneumonia often reported in trials. Another potential benefit of this short-duration method is that the speed of rewarming might not be as important when compared to longduration cooling. Very early clinical studies of long-duration cooling in stroke patients with large infarcts found that fast rewarming led to large elevations in intracranial pressure (ICP), which worsened outcome and was often fatal. This led to the development of gradual rewarming paradigms, which further extended the overall duration of cooling. Short duration cooling only maintains target temperature for a short period and may less likely result in rebound ICP. Previous work which has investigated hypothermia treatment for ICP elevation, using a similar rapid cooling and rewarming protocol as the ones described here, have shown no rebound ICP elevation after rewarming 23,24 .

Clinical trials of hypothermia for ischemic stroke treatment have not been able to translate the benefits of hypothermia that are reported in experimental studies. The mismatch in cooling rates and duration between experimental models and patients, are important variables that may account for this discrepancy. Having an experimental model of hypothermia that better resembles the clinical rate of cooling will allow for a more informed investigation into the benefits of hypothermia as a treatment measure for stroke patients. 


\section{Disclosures}

The authors have nothing to disclose.

\section{Acknowledgments}

This project was funded by the University of Newcastle, Hunter Medical Research Institute (HMRI) Dalara Early Research Career Researcher Fellowship, NSW Health EarlyMid Career Research Fellowship, and National Health and Medical Research Council (NHMRC) Australia.

\section{References}

1. Kurisu, K., Yenari, M. A. Therapeutic hypothermia for ischemic stroke; pathophysiology and future promise. Neuropharmacology. 134 (Pt B) 302-309 (2018).

2. Polderman, K. H. Induced hypothermia and fever control for prevention and treatment of neurological injuries. Lancet. 371 (9628), 1955-1969 (2008).

3. van der Worp, H. B., Sena, E. S., Donnan, G. A., Howells, D. W., Macleod, M. R. Hypothermia in animal models of acute ischaemic stroke: a systematic review and metaanalysis. Brain. 130 (Pt 12), 3063-3074 (2007).

4. Thomé, C., Schubert, G. A., Schilling, L. Hypothermia as a neuroprotective strategy in subarachnoid hemorrhage: a pathophysiological review focusing on the acute phase. Neurological Research. 27 (3), 229-237 (2005).

5. Mclntyre, L. A., Fergusson, D. A., Hébert, P. C., Moher, D., Hutchison, J. S. Prolonged therapeutic hypothermia after traumatic brain injury in adults: a systematic review. Journal of the American Medical Association. 289 (22), 2992-2999 (2003).

6. Kuczynski, A. M., Demchuk, A. M., Almekhlafi, M. A. Therapeutic hypothermia: Applications in adults with acute ischemic stroke. Brain Circulation. 5 (2), 43-54 (2019).

7. Shankaran, S. et al. Whole-body hypothermia for neonates with hypoxic-ischemic encephalopathy. New England Journal of Medicine. 353 (15), 1574-1584 (2005).

8. Jacobs, S. E. et al. Whole-body hypothermia for term and near-term newborns with hypoxic-ischemic encephalopathy: a randomized controlled trial. Archives of Pediatrics and Adolescent Medicine. 165 (8), 692-700 (2011).

9. Lyden, P. et al. Results of the ICTuS 2 Trial (Intravascular cooling in the treatment of stroke 2). Stroke. 47 (12), 2888-2895 (2016).

10. van der Worp, H. B. et al. Therapeutic hypothermia for acute ischaemic stroke. Results of a European multicentre, randomised, phase III clinical trial. European Stroke Journal. 4 (3), 254-262 (2019).

11. Wu, T. C., Grotta, J. C. Hypothermia for acute ischaemic stroke. Lancet Neurology. 12 (3), 275-284 (2013).

12. Hemmen, T. M. et al. Intravenous thrombolysis plus hypothermia for acute treatment of ischemic stroke (ICTuS-L): final results. Stroke. 41 (10), 2265-2270 (2010).

13. Lyden, P., Ernstrom, K., Raman, R. Determinants of pneumonia risk during endovascular hypothermia. Therapeutic Hypothermia and Temperature Management. 3 (1), 24-27 (2013).

14. van der Worp, H. B. et al. EuroHYP-1: European multicenter, randomized, phase III clinical trial of therapeutic hypothermia plus best medical treatment vs. 
best medical treatment alone for acute ischemic stroke. International Journal of Stroke. 9 (5), 642-645 (2014).

15. Lamb, J. A., Rajput, P. S., Lyden, P. D. Novel method for inducing rapid, controllable therapeutic hypothermia in rats using a perivascular implanted closed-loop cooling circuit. Journal of Neuroscience Methods. 267, 55-61 (2016).

16. Dumitrascu, O. M., Lamb J., Lyden, P. D. Still cooling after all these years: Meta-analysis od pre-clinical trials of therapeutic hypothermia for acute ischemic stroke. Journal of Cerebral Blood Flow and Metabolism. 36 (7), 1157-1164 (2016).

17. Wu, L. et al. Hypothermia neuroprotection against ischemic stroke: The 2019 update. Journal of Cerebral Blood Flow and Metabolism. 40 (3), 461-481 (2020).

18. Hemmen, T. M., Lyden, P. D. Hypothermia after acute ischemic stroke. Journal of Neurotrauma. 26 (3), 387-391 (2009).

19. Colbourne, F., Sutherland, G. R., Auer, R. N. An automated system for regulating brain temperature in awake and freely moving rodents. Journal of Neuroscience Methods. 67 (2), 185-190 (1996).

20. Campbell, K., Meloni, B. P., Zhu, H., Knuckey, N. W. Magnesium treatment and spontaneous mild hypothermia after transient focal cerebral ischemia in the rat. Brain Research Bulletin. 77 (5), 320-322 (2008).

21. Jespersen, B., Knupp, L., Northcott, C. A. Femoral arterial and venous catheterization for blood sampling, drug administration and conscious blood pressure and heart rate measurements. Journal of Visualized Experiments. (59), e3496 (2012).
22. Trotman-Lucas, M., Kelly, M. E., Janus, J., Gibson, C. L. Middle cerebral artery occlusion allowing reperfusion via common carotid artery repair in mice. Journal of Visualized Experiments. (143), e58191 (2019).

23. Murtha, L. A. et al. Short-duration hypothermia after ischemic stroke prevents delayed intracranial pressure rise. International Journal of Stroke. 9, 553-559 (2014).

24. Murtha, L. A. et al. Intracranial pressure elevation after ischemic stroke in rats: cerebral edema is not the only cause, and short-duration mild hypothermia is a highly effective preventative therapy. Journal of Cerebral Blood Flow and Metabolism. 35 (4), 592-600 (2015). 